\title{
A novel technique for endoscope progression in gastroscopy resection: " 6 "- turn of gastroscopy along the greater curvature wall for dissection of stromal tumor in the muscularis propria of the gastric fundus
}

Lu Liu ( $\square$ liulucmu4@126.com )

The Fourth Affiliated Hospital of China Medical University

Hai-Mei Guo

The Fourth Affiliated Hospital of China Medical University

\section{Ying Sun}

The Fourth Affiliated Hospital of China Medical University

\section{Shuang Cai}

The Fourth Affiliated Hospital of China Medical University

\section{Feng Miao}

The Fourth Affiliated Hospital of China Medical University

\section{Yan Zheng}

The Fourth Affiliated Hospital of China Medical University

\section{Yang Yu}

The Fourth Affiliated Hospital of China Medical University

\section{Zhi-Feng Zhao}

The Fourth Affiliated Hospital of China Medical University

\section{Research Article}

Keywords: Gastrointestinal Stromal Tumor, "6"-turn of gastroscopy along the gastric body wall, muscularis propria, gastric fundus

Posted Date: March 9th, 2022

DOI: https://doi.org/10.21203/rs.3.rs-734977/v2

License: (c) (i) This work is licensed under a Creative Commons Attribution 4.0 International License. Read Full License 


\section{Abstract}

Objective: The fundus of the stomach is a challenging region for endoscopic resection of gastrointestinal stromal tumors (GISTs), especially in the anterior wall of the fornix at the side of the greater curvature. The aim of this study was to introduce using the "6"-turn of gastroscopy along the greater curvature wall (6TGS) technique in operation on the gastric fundus and provide evidence of its advantages.

Methods: The clinical data of patients with stromal tumor in muscularis propria at the gastric fundus (STMF) at the Fourth Hospital of China Medical University between July 2016 and Dec 2020 were reviewed. The novel 6TGS technique was used in the procedure. The beneficial effects, suitability, applicable lesion site, influencing factors, and success rates of 6TGS were analyzed.

Results: Ten cases in total were reviewed, and the 6TGS technique was successfully performed in 7 cases (70\%). In all 7 patients, the gastroscope's tip could access the location just below the gastric cardia, and all angles/sites of the fundus of the stomach could be operated on. The lesion was easily accessed, and the gastroscope was stable with good left-right movement ability and moderate forward and backward movement ability.

Conclusion: 6TGS can improve the safety of gastroscopy resection of STMF and facilitate the excision of tumors in challenging sites in the gastric fundus. Patients need to be under general anesthesia with endotracheal intubation in the supine position to perform the 6TGS technique after distending the stomach with less air.

\section{Introduction}

Gastrointestinal stromal tumors (GISTs) are the most common gastrointestinal mesenchymal neoplasms and one of the clinically common submucosal tumors (SMT) of gastric origin ${ }^{1,2}$. Stromal tumors in muscularis propria (STMF) occur more at the fundus of the stomach, accounting for $51.5 \%$ of all GISTs sites $^{3,4}$. Notably, the clinical presentation of STMF is nonspecific, and few patients are presented with gastrointestinal bleeding ${ }^{5}$. Complete resection of STMF is currently the best treatment ${ }^{6}$, including different approaches such as traditional open surgery, laparoscopy, and gastroscopy dissection. However, many reports have demonstrated that gastroscopy dissection is the safest and most effective treatment for STMF of the fundus ${ }^{3}$.

Several gastroscopy dissection techniques are used nowadays in clinical practice, including Endoscopic submucosal dissection (ESD) ${ }^{7}$, endoscopic submucosal excavation (ESE) ${ }^{8}$, endoscopic full-thickness resection (EFTR) ${ }^{9,10}$, submucosal tunneling endoscopic resection (STER) ${ }^{11}$, and the combination of EFTR and laparoscopic approach $3,12-15$. The fundus of the stomach is considered a challenging area for endoscopic resection of tumors in muscularis propria ${ }^{16}$, especially when using ESD ${ }^{17}$, ESE, or EFTR ${ }^{18,19}$. Additionally, endoscopic resection of lesions in the middle of the fornix and the anterior wall of the fornix 
is extremely difficult in clinical practice, especially the anterior wall of the fornix at the side of the greater curvature.

IN some STMF cases, the removal procedure requires the U-turn of the distal tip of the gastroscope ${ }^{20}$. In these cases, the progress of the tip is opposite to the moving direction of observing, and the body of the gastroscopy becomes suspended without support; thus, it is difficult to operate and control. In 2016, Professor Zhi-Feng Zhao discovered the " 6 "-turn of gastroscopy along the greater curvature wall (6TGS) during the endoscopic treatment for a patient with STMF and successfully completed the procedure. Using 6TGS, the endoscopy progresses into the stomach continuously so that the tip of the gastroscopy makes a "6"-turn along the gastric wall and accesses the gastric fundus without access to the antral cavity. The present study introduces this new endoscopy progression technique and the results of its applicability in practice.

\section{Discussion}

\section{The 6TGS technique is a novel direct endoscopy progression method, with no paradoxical movement involved.}

Our team discovered the 6TGS technique during an STMF procedure. Through practice and after analyzing the results, the present study summarizes the operation method and the success rate of 6TGS. Unlike the typical progression way, in which the gastroscope's tip usually reaches the antrum first, the 6TGS technique uses the resistance of the posterior wall of the upper gastric body (near the junction with the fundus) and the greater curvature as the supporting force to progress the gastroscope along the arc from the posterior wall of the gastric body to the angular incisure, making a " 6 "-turn along the gastric wall. This route allows the gastroscope to access the fundus of the stomach and even access just below the gastric cardia. The fundus of the stomach can then be handled from all angles. Thus, there is no need for paradoxical movement, making it much easier for the operators to access the lesion for more visualization and handling in operation. This novel endoscopy progression method is guaranteed to improve the STMF operation.

Additionally, when observing the fundus of the stomach using the 6TGS technique, it was found that the mucosa just below the gastric cardia can be clearly visualized. The gastroscope tip could operate the lesion at a close distance, which can significantly benefit operating on lesions below the gastric cardia.

\section{TGS: Solutions to the challenges of endoscopic treatment of GIST in the gastric fundus}

The fundus of the stomach is considered a challenging area for ESD ${ }^{17}$, ESE, or EFTR ${ }^{18,19}$; the ESD technique is complex and prone to perforation ${ }^{21}$, and the EFTR technique intentionally destroys the full 
thickness of the gastric wall, causing perforation to remove the lesion then sutures the wound by nylon suture and or hemostatic clips ${ }^{22}$. One of the current endoscopic treatments used for STMF is EFTR ${ }^{16}$. And indeed, EFTR surgery was predominant in our surgeries (5/7).

Endoscopic operation on gastric fundus is challenging, mainly due to the need for the traditional endoscopic progression method to be bent backward (U-turn) to access and observe the fundus of the stomach completely. The direction of endoscopy body movement is opposite to the direction of observation (paradoxical movement), and the endoscopy body gets suspended, which makes the operating space for the fundus of the stomach limited ${ }^{17}$. When the endoscope's body is suspended using the common U-turn technique, the forward injection force becomes immensely impaired, and the injection needle is perpendicular to the muscularis propria, making it difficult to find the submucosal space. Additionally, the resistance increases when surgical instruments (such as electrotome, thermal coagulation forceps, etc.) are delivered through the instrument channel due to the big curved angle. Following the current commonly used technique, we found that endoscopic treatment of STMF lesions in the middle of the fornix and anterior wall of the fornix was extremely challenging, especially the anterior wall of the fornix at the side of the greater curvature. Thus, a better endoscopic technique was needed to observe, deliver surgical instruments, and operate on these challenging tumor sites.

\section{Operating Characteristics of the 6TGS}

6TGS solves the problem of eliminating the need for paradoxical movement; thus, the gastroscope's body becomes stable, contributing to more exposure of the lesion, improving the visualization, and increasing the forward puncture ability of the injection needle during submucosal injection.

Except for STMF in the posterior wall of the fundus, 6TGS allows the angle of the endoscope's tip to reach the mucosa and muscle layer for submucosal injection, and a transverse incision can be used to cut the mucosa above the lesion, as each layer below the mucosa/above the lesion could be clearly observed. 6TGS ensures that the direction of the electrical cutting tool is parallel to the muscle during dissection, which reduces injury and the risk of bleeding.

\section{Factors affecting 6TGS}

The success rate of 6TGS was 70\% in our clinical practice. At present, 6TGS could only be performed while patients were in the supine position and under general anesthesia with endotracheal intubation. The 6TGS failure in 3 cases may be related to the morphology of the gastric fundus. In all successful cases, less air volume in the stomach was required. More case studies are needed to investigate the factors influencing the success rate of 6TGS. The success rate of 6TGS may be related to the morphology of the fundus, the amount of air in the stomach, and the patient posture after general anesthesia, which requires further observation and study in the future. The present study has some limitations due to the small sample of STMF patients included who qualified for 6TGS; thus, bigger observational studies are needed to promote 6TGS in the future. 


\section{Conclusions}

The novel 6TGS technique can improve the safety of gastroscopy resection of STMF by providing more exposure to the lesion, improving the visualization, and increasing the injection needle's forward puncture ability during submucosal injection without the need for paradoxical movement in the currently challenging STMF tumor sites in the gastric fundus. The 6TGS technique is recommended to be performed while the patients were in the supine position, under general anesthesia with endotracheal intubation, and with a small amount of air inflation.

\section{Patients And Methods}

This study is approved by IRB of our hospital. All methods were carried out in accordance with the Declaration of Helsinki. The informed consent was obtained from all subjects.

\section{Subjects}

The clinical data of STMF patients who accepted the new 6TGS technique at the Fourth Hospital of China Medical University between July 2016 and Dec 2020 were retrospectively analyzed. Since it is a retrospective study, not a clinical trial, no IRB approval or informed consent are needed.

Case selection requirements: (i) gastroscopy revealed elevated mucosa at the fundus of the stomach; (ii) endoscopic ultrasonography revealed STMF; (iii) no history of gastric cardiac or gastric surgery; (iv)

tolerance to general anesthesia and tracheal intubation; (v) the diameter of the lesions was $<5 \mathrm{~cm}^{23}$; (vi) patients who were informed with the new endoscopic technique and signed the consent form.

Exclusion Criteria (contraindications for endoscopic resection of gastrointestinal submucosal tumors ${ }^{23}$ ): (i) an enlarged lymph node or distant metastasis lesion was identified; (ii) patients with a poor general health condition who couldn't tolerate endoscopic surgery; (iii) patients with bleeding and/or ulceration on the surface of the lesion.

\section{Methods}

The endoscopy progression technique

The presented endoscopy progression technique for gastric fundus surgery involves using the " 6 "-turn of the gastroscope along the greater curvature wall. The gastroscope does not enter the gastric antrum after passing through the gastric cardia while the endoscopy progresses continuously; the gastroscope body then makes a "6"-turn along the wall of the posterior wall of the upper gastric body and the wall of greater curvature to access the fornix of the stomach. When the gastroscope pushes forward, its tip moves towards the fundus of the stomach, and when the gastroscope pulls back, its tip moves away from the fundus. Thus, the gastroscope body is fixed in the upper gastric body by the stomach wall without being suspended. We named this endoscopy progression technique: " 6 "-turn of gastroscopy along the greater 
curvature wall (6TGS). This new technique avoided the paradoxical movement in other advanced endoscopy progression skills such as common U-turn. (Please refer to Fig. 1).

\section{Endoscopic resection}

All endoscopic surgeries were performed following Professor Zhi-Feng Zhao's guidance of 6TGS. The operators were senior endoscopists in our endoscopic treatment center who had several years of ESD surgery experience. The patients underwent endotracheal intubation under intravenous anesthesia before the endoscopic surgery. $\mathrm{CO} 2$ was the anesthesia of choice for the endoscopic surgeries of STMF 24,25 .

For the endoscopic resection of STMF, we mainly used $\mathrm{ESD}^{26}$ or $\mathrm{EFTR}^{27}$, depending on the growth pattern and depth of invasion of the tumor.

\section{Data collection}

The following data were collected and analyzed: (i) basic information of patients; (ii) whether 6TGS was successfully performed; (iii) the selected endoscopic method (after successful 6TGS). Lesion sites suitable for 6TGS were selected according to the division of gastric fundus and the lesion area, as shown in Fig. 2. The success rate, results, operating characteristics, and success and failure factors of 6TGS were analyzed. The operating characteristic assessment was scored using the following grades: very good, good, fair, and poor.

\section{Postoperative Management}

After the ESD procedure, patients were required bed rest, food fasting and water fasting for three days, and administration of antibiotics for three days. The above measures and gastrointestinal decompression were performed for five days after EFTR. Parenteral nutrition was given during fasting, and continuous oral proton pump inhibitor and gastric mucosal protective agents were administered 6-8 weeks postoperatively. Patients were observed for complications of ESD and EFTR, mainly bleeding and perforation ${ }^{28}$.

\section{Follow-Up}

All patients were followed up regularly with gastroscopy examination at 3, 6, and 12 months after the operation, and then were examined once a year to observe the wound healing and check for any residual or recurrent tumors.

\section{Histopathological Evaluation}

The resected pathological specimens were immediately fixed in $10 \%$ neutral buffered formalin, followed by histopathological examination. A pathologist confirmed the diagnosis, and immunohistochemical staining was performed if necessary for differential diagnosis.

\section{Statistical Analyses}


Statistical analyses were performed using IBM SPSS Statistics 25.0 (SPSS Inc., Chicago, IL, USA). Continuous data were expressed as $₫ X \pm s$. Categorical data were presented as numbers or percentages (\%).

\section{Results}

\section{Patient Information}

A total of 112 cases diagnosed with STMF and treated in the Fourth Affiliated Hospital of China Medical University from 2016 to 2020 were evaluated. There were 10 patients qualified for the 6TGS technique (2 males and 8 females), aged $57.6 \pm 10.07$ (46-78), with a lesion diameter of $1.34 \pm 1.63(0.5-5.0) \mathrm{cm}$. 6TGS was successfully performed in 7 patients (70\%), failed in 3 patients (30\%). Endoscopic treatment methods after successful 6TGS: EFTR: 5 cases; ESD: 2 cases. (See Table 1). 
Table 1

Case Information

\begin{tabular}{|lll|}
\hline Items & Details & $\begin{array}{c}\text { Number of } \\
\text { cases }\end{array}$ \\
\hline Age & $57.6 \pm 10.07(46-78)$ years & 10 \\
\hline Sex & Male & 2 \\
\hline Lesion diameter & Female & 8 \\
\hline Location & $1.34 \pm 1.63(0.5-5.0) \mathrm{cm}$ & 1 \\
\hline & Middle of the fornix & 4 \\
\hline & Great curved side of anterior wall of & 4 \\
\hline the fornix & Posterior wall of fundus & 1 \\
\hline Greater curvature side of fundus & 3 \\
\hline Endoscopic surgery method after & Greater curvature side of middle of the & 3 \\
\hline fornix & ESD & 2 \\
\hline Successful 6TGS & EFTR & 5 \\
\hline Surgical duration: & $7 / 10$ & $70 \%$ \\
\hline Complications: & $83.86 \pm 46.74$ (47-182) min & 0 \\
\hline Length of hospitalization & None observed & \\
\hline
\end{tabular}

\section{The results of 6TGS application:}

After 6TGS was used in 10 patients, the tip of the gastroscope could access the location just below the gastric cardia in 7 patients. All angles of the fundus of the stomach were operable in all 7 patients. Using 6TGS, there was no need for paradoxical movement; it was much easier to access the lesion and observe.

\section{The Suitable location for 6TGS:}

6TGS allowed clear visualization of the lesions in all the fundus area and the mucosa just below the gastric cardia. 


\section{Operating characteristics of 6TGS:}

In this study, 6TGS was mainly used for submucosal injection and lesion dissection during endoscopic treatment of STMF. Post-resection trauma suturing was performed in a conventional endoscopy progression.

Using 6TGS, we were able to access and visualize the following area in the lesion: the edge close to the pylorus (7/7), the edge close to gastric cardia (5/7), and the left and right edges (7/7). When the lesion was large, it was difficult for the gastroscopy body to cross the lesion to observe the lesion's edge at the gastric cardia.

As for the Operational Performance Evaluation of the 6TGS technique, it was very accessible to navigate the tip of the gastroscope tip left or right using the small adjusting knobs; however, moving the gastroscope forward and backward was still of moderate difficulty. The gastroscope's body makes a "6"turn along the gastric wall, which made the gastroscopy tip stable at every angle due to the support of the gastric wall. The gastroscope was stable at every angle without the need for an assistant to support the endoscope, and the operator could manually adjust the devices in the instrument channel to complete a variety of complex adjustments, cutting, and hemostasis. Since the gastroscopy tip could access the lesion's edges with stable visualization, the details of each layer below the mucosa and above the lesion could be clearly observed; however, water accumulation in the lesion area had certain interference. The stability of the gastroscope body increased the forward puncture ability of the injection needle during submucosal injection. The 6TGS technique also allowed the gastroscopy body to form a small injection inclination angle with the gastric wall, making it easier to enter the submucosal space. (See Table 2.)

Table 2

Evaluation of 6TGS operating characteristics

\begin{tabular}{|lllll|}
\hline Items & Very good & Good & Fair & Poor \\
\hline The left and right movement ability & & $7 / 7$ & & \\
\hline Push forward and pull back ability & & & $7 / 7$ & \\
Ability to stay in place & $7 / 7$ & & \\
Visualization ability & $6 / 7$ & $1 / 7$ & \\
Submucosal injection result & $6 / 7$ & $1 / 7$ & \\
\hline
\end{tabular}

The dissector can be parallel to the muscularis propria forming a good inclination angle during the dissection of the STMF except for tumors at the posterior wall of the fundus. There was some resistance while progressing the instruments in the instrument channel using the 6TGS technique; however, this defect is the same as in the traditional U-turn. 


\section{Factors affecting the success of 6TGS.}

Surgeries were performed while the patients were in the supine position under general anesthesia with endotracheal intubation. In 3 patients, 6TGS could not be completed due to the fundus morphology of the lesion. 6TGS was successfully performed in 7 cases, in which the visualization was air-dependent, and the gastric inflation was successful with less air volume. Notably, 6TGS could not be completed with more air volume in the stomach.

\section{Postoperative and follow-up results:}

The operation duration using the 6TGS technique was $83.86 \pm 46.74(47-182) \mathrm{min}$, and the hospital stay was $7.29 \pm 0.49(7-8)$ days. Postoperative histopathological evaluation revealed stromal tumors. No complications were observed, and the patients recovered well after the operation. During the postoperative follow-ups, there was no residual or recurrent tumor, metastasis, or death.

\section{Declarations}

\section{Conflicts of Interest}

All authors (Hai-Mei Guo, Ying Sun, Shuang Cai, Feng Miao, Yan Zheng, Yang Yu, Zhi-Feng Zhao, Lu Liu) declare that they have no conflict of interest.

\section{References}

1. Poveda, A.et al. GEIS guidelines for gastrointestinal sarcomas (GIST). Cancer Treat Rev 55, 107-119, doi:10.1016/j.ctrv.2016.11.011 (2017).

2. Matsuda, T.et al. Laparoscopic and luminal endoscopic cooperative surgery can be a standard treatment for submucosal tumors of the stomach: a retrospective multicenter study. Endoscopy 49, 476-483, doi:10.1055/s-0043-104526 (2017).

3. Guo, Y.et al. Endoscopic Removal of Gastrointestinal Stromal Tumors in the Stomach: A SingleCenter Experience. Gastroenterol Res Pract 2019, 3087298, doi:10.1155/2019/3087298 (2019).

4. Hou, Q.et al. [Analysis of gastric gastrointestinal stromal tumors in Shandong Province: a midterm report of multicenter GISSG1201 study]. Zhonghua Wei Chang Wai Ke Za Zhi 20, 1025-1030 (2017).

5. Pih, G. Y.et al. Clinical outcomes of upper gastrointestinal bleeding in patients with gastric gastrointestinal stromal tumor. Surg Endosc 34, 696-706, doi:10.1007/s00464-019-06816-9 (2020).

6. Sanchez-Hidalgo, J. M.et al. Gastrointestinal stromal tumors: A multidisciplinary challenge. World J Gastroenterol 24, 1925-1941, doi:10.3748/wjg.v24.i18.1925 (2018).

7. Li, L.et al. Endoscopic submucosal dissection of gastric fundus subepithelial tumors originating from the muscularis propria. Exp Ther Med 6, 391-395, doi:10.3892/etm.2013.1181 (2013). 
8. Shi, W. B.et al. Comparison between air and carbon dioxide insufflation in the endoscopic submucosal excavation of gastrointestinal stromal tumors. World J Gastroentero/ 18, 7296-7301, doi:10.3748/wjg.v18.i48.7296 (2012).

9. Li, J., Meng, Y., Ye, S., Wang, P. \& Liu, F. Usefulness of the thread-traction method in endoscopic fullthickness resection for gastric submucosal tumor: a comparative study. Surg Endosc 33, 2880-2885, doi:10.1007/s00464-018-6585-2 (2019).

10. Andalib, I., Yeoun, D., Reddy, R., Xie, S. \& Iqbal, S. Endoscopic resection of gastric gastrointestinal stromal tumors originating from the muscularis propria layer in North America: methods and feasibility data. Surg Endosc 32, 1787-1792, doi:10.1007/s00464-017-5862-9 (2018).

11. Liu, L.et al. Endoscopic Submucosal Dissection of the Angiolipoma at Hypopharynx-Esophageal Introitus. Gastroenterol Res Pract 2020, 3581267, doi:10.1155/2020/3581267 (2020).

12. Fernandes, J.et al. Hybrid NOTES: Complete Endoscopic Resection of the Gastric Wall Assisted by Laparoscopy in a Gastric Fundus Gastrointestinal Stromal Tumor. GE Port J Gastroentero/ 26, 215217, doi:10.1159/000491709 (2019).

13. Aisu, Y., Yasukawa, D., Kimura, Y. \& Hori, T. Laparoscopic and endoscopic cooperative surgery for gastric tumors: Perspective for actual practice and oncological benefits. World J Gastrointest Oncol 10, 381-397, doi:10.4251/wjgo.v10.i11.381 (2018).

14. Kadkhodayan, K., Rafiq, E. \& Hawes, R. H. Endoscopic Evaluation and Management of Gastric Stromal Tumors. Curr Treat Options Gastroenterol 15, 691-700, doi:10.1007/s11938-017-0160-0 (2017).

15. Matsumoto, S.et al. Non-exposed endoscopic wall-inversion surgery for pediatric gastrointestinal stromal tumor: A case report. Asian J Endosc Surg 12, 322-325, doi:10.1111/ases.12641 (2019).

16. Ge, N., Hu, J. L., Yang, F., Yang, F. \& Sun, S. Y. Endoscopic full-thickness resection for treating small tumors originating from the muscularis propria in the gastric fundus: An improvement in technique over 15 years. World J Gastrointest Oncol 11, 1054-1064, doi:10.4251/wjgo.v11.i11.1054 (2019).

17. Wen, Z. Q.et al. Application of blunt dissection in ESD of a gastric submucosal tumor. World J Gastroenterol 20, 6698-6700, doi:10.3748/wjg.v20.i21.6698 (2014).

18. Li, B.et al. Efficacy and safety of endoscopic resection for small submucosal tumors originating from the muscularis propria layer in the gastric fundus. Surg Endosc 33, 2553-2561, doi:10.1007/s00464018-6549-6 (2019).

19. Shi, Q.et al. Clinical Values of Dental Floss Traction Assistance in Endoscopic Full-Thickness Resection for Submucosal Tumors Originating from the Muscularis Propria Layer in the Gastric Fundus. J Laparoendosc Adv Surg Tech A 28, 1261-1265, doi:10.1089/lap.2018.0030 (2018).

20. Lee, S. H., Park, Y. K., Cho, S. M., Kang, J. K. \& Lee, D. J. Technical skills and training of upper gastrointestinal endoscopy for new beginners. World J Gastroenterol 21, 759-785, doi:10.3748/wjg.v21.i3.759 (2015).

21. Nishizawa, T. \& Yahagi, N. Endoscopic mucosal resection and endoscopic submucosal dissection: technique and new directions. Curr Opin Gastroentero/33, 315-319, 
doi:10.1097/MOG.0000000000000388 (2017).

22. Huang, J.et al. Endoscopic full-thickness resection for gastric gastrointestinal stromal tumor originating from the muscularis propria. Rev Assoc Med Bras (1992) 64, 1002-1006, doi:10.1590/1806-9282.64.11.1002 (2018).

23. Zhou, P., Zhong, Y. \& Li, Q. [Chinese Consensus on Endoscopic Diagnosis and Management of Gastrointestinal Submucosal Tumor(Version 2018)]. Zhonghua Wei Chang Wai Ke Za Zhi 21, 841852 (2018).

24. Li, X., Dong, H., Zhang, Y. \& Zhang, G. CO2 insufflation versus air insufflation for endoscopic submucosal dissection: A meta-analysis of randomized controlled trials. PLoS One 12, e0177909, doi:10.1371/journal.pone.0177909 (2017).

25. Takada, J.et al. Safety of Carbon Dioxide Insufflation during Endoscopic Submucosal Dissection for Esophageal Squamous Cell Carcinoma. Dig Dis 37, 93-99, doi:10.1159/000492870 (2019).

26. An, W.et al. Endoscopic submucosal dissection for gastric gastrointestinal stromal tumors: a retrospective cohort study. Surg Endosc 31, 4522-4531, doi:10.1007/s00464-017-5511-3 (2017).

27. Tan, Y.et al. Comparison between submucosal tunneling endoscopic resection and endoscopic fullthickness resection for gastric stromal tumors originating from the muscularis propria layer. Surg Endosc 31, 3376-3382, doi:10.1007/s00464-016-5350-7 (2017).

28. Akahoshi, K., Oya, M., Koga, T. \& Shiratsuchi, Y. Current clinical management of gastrointestinal stromal tumor. World J Gastroenterol 24, 2806-2817, doi:10.3748/wjg.v24.i26.2806 (2018).

\section{Figures}

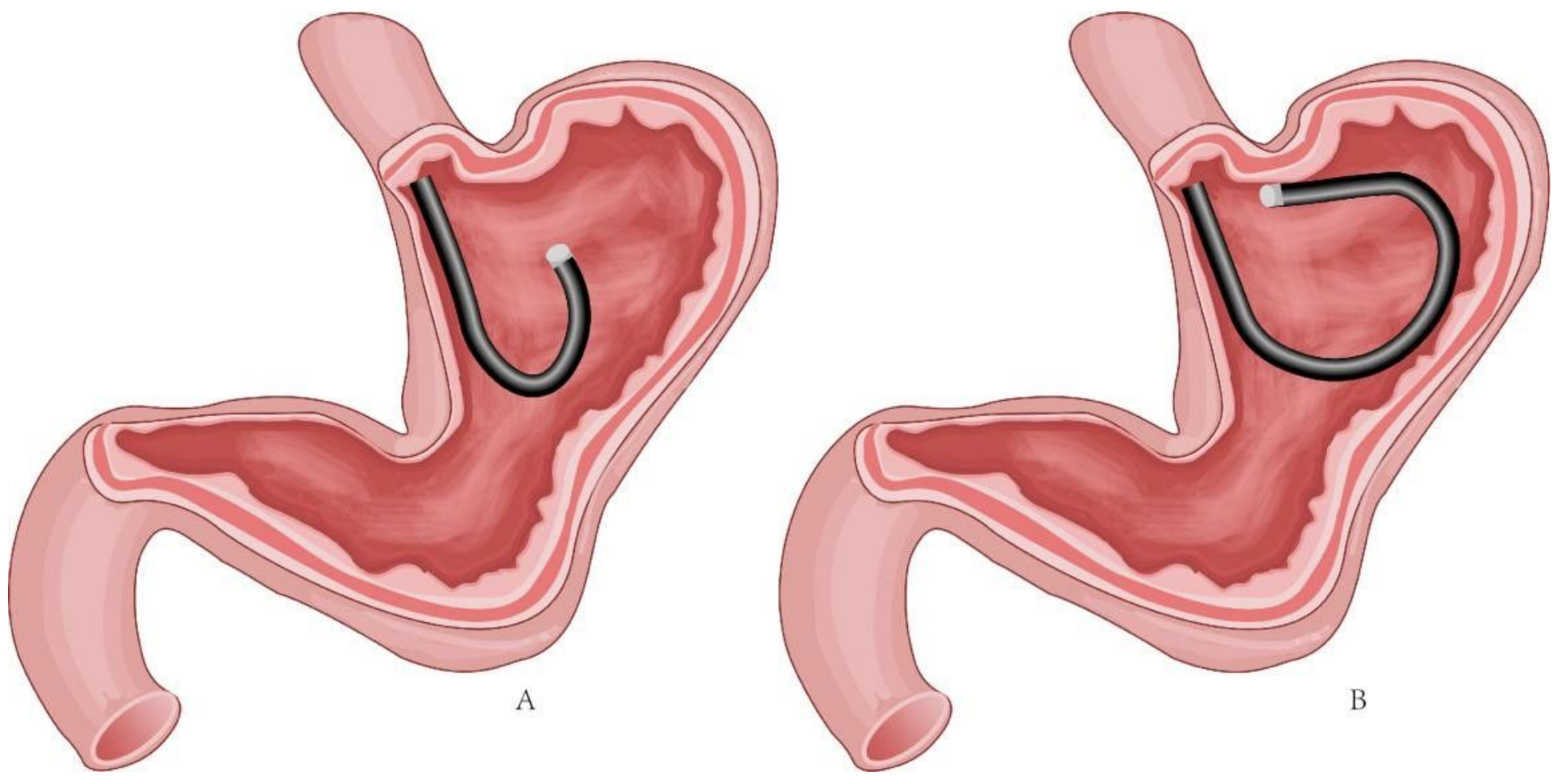


$A \llbracket G a s t r i c$ fundus and cardia were observed by conventional U-turn technique, the gastroscopy body is suspended, without support. B囚Endoscopy progression just below the gastric cardia using the "6"-turn of gastroscopy along the greater curvature wall (6TGS) technique, the the gastroscopy body is supported by the gastric wall.

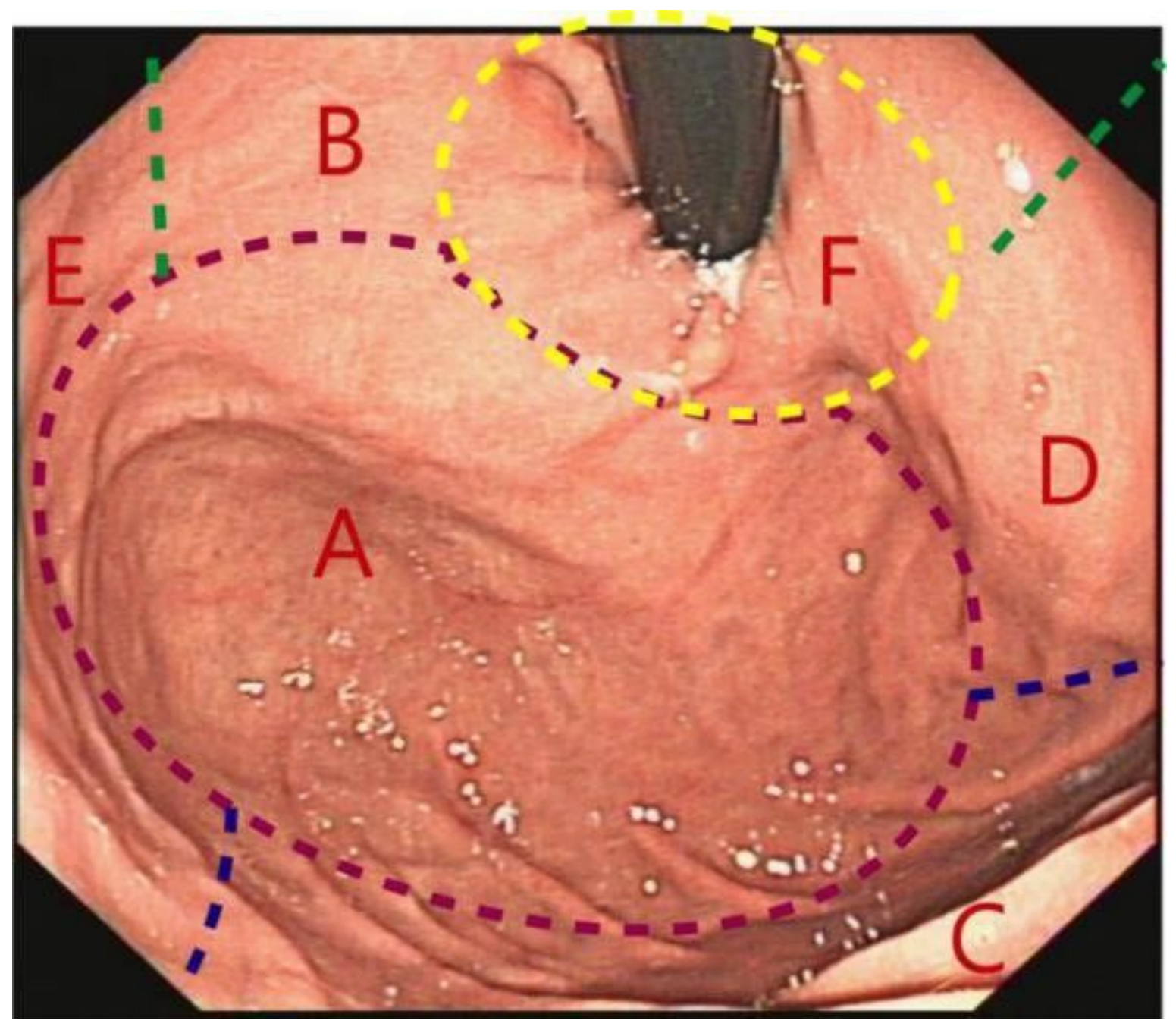

Figure 2

Gastric Fundus Subdivisions: A: Fornix; B: Lesser curvature of the stomach; C: Greater curvature of the stomach; D: Anterior wall; E: Posterior wall; F: Gastric cardia

\section{Supplementary Files}

This is a list of supplementary files associated with this preprint. Click to download.

- video1.mp4 